\title{
EDITORIAL
}

\section{¿VALORAR LA INVESTIGACIÓN Y LA CALIDAD DE LA PRODUCCIÓN CIENTÍFICA SÍ ES PERTINENTE?}

\author{
Germán Anzola Montero \\ Rector
}

Durante más de dos décadas, COLCIENCIAS lidera el Sistema Nacional de Ciencia, Tecnología e Innovación, enfocando sus acciones en cuatro áreas específicas: Educación para la Investigación; Investigación; Innovación y Mentalidad y Cultura Científica. Para el caso específico de la Investigación, se ha definido que es la dimensión que promueve la generación de conocimiento y, con ello, pretende fortalecer "las capacidades de la investigación y el desarrollo" y, para lograr tal fin, abre convocatorias, que persiguen objetivos como "contar con información actualizada sobre los Grupos de Investigación, Desarrollo Tecnológico o de Innovación y los Investigadores del país, sus actividades y los resultados logrados para generar conocimiento sobre las capacidades, fortalezas, debilidades y potencialidades de quienes integran el Sistema Nacional de CTel" (COLCIENCIAS, 2017a).

Si ello se cumpliera, los resultados de la última Convocatoria Nacional para el Reconocimiento y Medición de Grupos de Investigación, Desarrollo Tecnológico o de Innovación y para el Reconocimiento de Investigadores del SNCTel-2015 -No. 737-, no arrojaría tan escandalosos resultados; por ejemplo, aplicaron más de 66 mil hojas de vida registradas y certificadas en la Plataforma ScienTI Colombia, en los aplicativos CvLAC (currículos), avaladas por las instituciones correspondientes y solamente COLCIENCIAS reconoció a un poco más de 10 mil investigadores, con las siguientes vigencias: Investigador Senior: 1.218, tres años de vigencia, hasta mayo de 2019; Investigador Asociado: 2.767, dos años, hasta Mayo 21 de 2018 e Investigador Junior: 6.057, un año, hasta Mayo 21 de 2017. Estas cifras corresponden a todo el país, lo que indica que solamente el $15 \%$ de profesionales tienen la capacidad para solucionar los problemas nacionales y de sus pobladores. Y, cel resto?, cino son igual de competentes? No se debe desconocer que las Universidades invierten grandes sumas de dinero para formar a sus profesores e investigadores (COLCIENCIAS, 2016a).

Ahora bien, con los grupos de investigación la situación no es nada alentadora. Este mismo Modelo de Medición fijó como objetivos:
- Proveer, a la comunidad interesada en temas de CTeI, información actualizada acerca de las actividades desarrolladas por los grupos de investigación, desarrollo tecnológico o de innovación nacionales y de sus investigadores.

- Establecer las capacidades en investigación, desarrollo tecnológico, innovación, formación de talento humano y apropiación social del conocimiento que tiene la comunidad dedicada a CTeI, en el país.

- Evaluar el potencial y el desempeño de los grupos investigación, desarrollo tecnológico, o de innovación, centros de investigación o de desarrollo tecnológico y demás entes del Sistema Nacional de CTeI que desarrollan investigación.

- Identificar el recurso humano involucrado en actividades de CTel en Colombia.

- Determinar cuáles son las líneas de investigación desarrolladas en el país y cuál ha sido su evolución.

- Diseñar, implementar y evaluar políticas públicas en CTeI.

Siendo así, los resultados arrojados por COLCIENCIAS (2016b), 5.796 registros de grupos -avalados por las instituciones-, estuvieron inscritos, donde solamente 4.458, cumplieron con las condiciones para reconocimiento, quedando su clasificación, así: Grupos A1: 408; Grupos A: 549; Grupos B: 952; Grupos C: 1.939 y Grupos D: 610, es decir, que el 76,9\% de todos los grupos de investigación del país, es decir las unidades de organización y gestión de la actividad investigadora, son idóneos para formular uno o varios problemas, trazar un plan estratégico y producir unos resultados de conocimiento sobre un tema en particular. Es oportuno preguntar: cel resto de proyectos que se formulan no son igual de significativos?, ¿no todos buscan la transferencia y la aplicación del conocimiento científico al país y a sus gentes?

La Universidad de Ciencias Aplicadas y Ambientales U.D.C.A, actualmente, cuenta 13 grupos de investigación, asociados a sus áreas de conocimiento: en Administración y Negocios: Compensación con justicia social; en Ciencias: Productos 
Naturales U.D.C.A; en Ciencias Ambientales: Grupo Red "Universidad y Ambiente" - GRUA (grupo interdisciplinar que hace parte Ciencias de la Educación) y Sostenibilidad Ambiental; en Ciencias de la Educación: Estilos de Pensamiento y Estilos de aprendizaje; en Ciencias de la Salud: Grupo de Investigaciones Biomédicas y de Genética Humana Aplicada - GIBGA, Saber y Practica en Enfermería, Cuidado de la Salud y Desarrollo Sostenible y Ciencias del Deporte y la Actividad Física; en Ciencias Pecuarias: Grupo de Investigación en Ciencia Animal; en Derecho, Ciencias Sociales y Humanas: Ucansuca y en Ingeniería: Producción agrícola sostenible e Investigaciones Geográficas para el Desarrollo Territorial.

Siendo coherentes con los objetivos propuestos por COLCIENCIAS, en materia de investigación, el PEI de la Universidad, en los lineamientos específicos para la investigación, reza: "En la U.D.C.A la investigación se define como un trabajo intelectual racional y crítico que, de manera rigurosa y sistemática, genera y aplica el conocimiento científico y tecnológico en un horizonte de pertinencia cultural y social, reconociendo su poder transformador y las implicaciones ético-políticas que conlleva. La finalidad de la investigación en la U.D.C.A es la generación y aplicación del conocimiento científico y tecnológico, el avance de las ciencias aplicadas y ambientales y la búsqueda de soluciones a problemas relevantes de la sociedad. La investigación, se realiza desde el respeto a los valores de la ciencia, la libertad de pensamiento, el reconocimiento de la pluralidad paradigmática, metodológica y procedimental para solucionar los problemas del conocimiento científico y el reconocimiento de sus fines como ciencia básica y aplicada" (PEI, 2013). Creo que, acertadamente, la Universidad apunta al horizonte que exige el país.

Teniendo en cuenta este panorama y dando cumplimiento cabal a lo estipulado por las diversas Convocatorias emitidas por COLCIENCIAS, se puede concluir que la situación es bastante preocupante, en especial, para aquellas Universidades, donde su fortaleza mayor es la docencia de alta calidad, pues fijan como norte la "formación de profesionales integrales en las diferentes áreas del conocimiento, personas con altos valores ciudadanos" (U.D.C.A, 2017) y logran, con éxito, combinar la docencia con la investigación. Si el país requiere y demanda de una investigación de alta calidad, de investigadores con un excelente potencial científico y que su proyección sea beneficiar a la población, es hora que las Universidades le demos una mirada a estas exigencias e, imperativamente, analicemos a dónde se dirigen los dineros del erario público, destinado para este propósito.

Es oportuno aclarar que estas Convocatorias también "permiten la financiación de programas o proyectos de CTel, con énfasis en aquellos que contribuyan a la solución de los problemas del país y sus regiones" (COLCIENCIAS, 2017b).

Son varias las convocatorias que el Estado asigna presupuesto, por ejemplo, la Convocatoria Ecosistema Científico para la conformación de un banco de programas de $\mathrm{I}+\mathrm{D}+\mathrm{i}$ elegibles, que contribuyan al mejoramiento de la calidad de las Instituciones de Educación Superior colombianas-2017, con una asignación de \$80 millones de pesos; la Convocatoria para proyectos de Ciencia, Tecnología e Innovación en Salud-2017, cuyo objetivo es contribuir a la solución de los retos en salud del país mediante la financiación de proyectos de investigación científica, desarrollo tecnológico e innovación de alto impacto, con una asignación de \$34.876.000.000 o la Convocatoria Nacional para la Conformación de un banco de proyectos elegibles de Generación de Nuevo Conocimiento - 2017, con \$27.510.200.516. Y cabe la pregunta: ¿̇por qué la gran mayoría de Universidades no tienen la posibilidad de "ganar" estas convocatorias y una minoría, sí?

Para el 2017, nuevamente debemos someternos a las pretensiones, por parte de COLCIENCIAS, emitidas en la Convocatoria Nacional para el Reconocimiento y Medición de Grupos de Investigación, Desarrollo Tecnológico o de Innovación y para el Reconocimiento de Investigadores del Sistema Nacional de Ciencia, Tecnología e Innovación - SNCTeI - 2017. ¿Qué les deparará a nuestros grupos de investigación y a nuestros docentes-investigadores?, ¿Seguirán las Universidades disponiendo de miles de millones de pesos de su presupuesto - un gran porcentaje de ellas, destinan estos recursos de las matrículas- para solventar sus necesidades en investigación?, ¿COLCIENCIAS si presta un buen servicio de influencia de la investigación colombiana, en el ámbito internacional?, ¿Seguirá siendo esta subjetividad una palanca para el reconocimiento de alta calidad?

A manera de conclusión es necesario enfatizar que las Universidades, hoy por hoy, se preocupan por desarrollar y mejorar su actividad investigativa, por varias razones: 1) para lograr el reconocimiento de sus investigadores y de sus grupos, a nivel nacional e internacional; 2) para pertenecer a excelentes redes sociales científicas, que les permita potenciar sus relaciones con otros profesionales y encontrar un espacio idóneo para el desarrollo de sus actividades de investigación; 3) para renovar sus registros calificados, gracias a las exigencias de las condiciones impuestas por COLCIENCIAS; 4) para dar cumplimiento a los procesos de acreditación institucional, entre otras.

Traigo a colación lo que señalaron Clemencia Bonilla y María Dolorez Pérez, del Centro de Estudios en Educación Superior: "Sobra decir que las instituciones de educación superior 
de docencia tienen la misión de formar profesionales de alto nivel para la economía, la política y la cultura y que están obligadas a nutrirse de la investigación y la innovación que produzcan las instituciones dedicadas a la investigación. Junto con las universidades de docencia con investigación pueden cumplir una función significativa en la extensión y proyección social" (Bonilla \& Pérez, 2017).

\section{BIBLIOGRAFÍA}

1. BONILLA O., C.; PÉREZ P., M.D. 2017. Actividad investigativa en las instituciones de educación superior: ¿se reconoce la diversidad institucional existente en el país? Disponible desde Internet en: http://www. universidad.edu.co/index.php/ensayos-acadcosmainmenu-81/14601-actividad-investigativaen-las-instituciones-de-educacion-superior-se-reconoce-la-diversidad-institucional-existente-en-el-pais (con acceso 22/05/2017).

2. COLCIENCIAS. 2017a. Convocatoria nacional para el reconocimiento y medición de Grupos de Investigación, Desarrollo Tecnológico o de Innovación y para el reconocimiento de Investigadores del Sistema Nacional de Ciencia, Tecnología e Innovación. Disponible desde Internet en: http://colciencias.gov.co/ convocatorias/investigacion/convocatoria-nacionalpara-el-reconocimiento-y-medicion-grupos (con acceso 22/05/2017).
3. COLCIENCIAS. 2017b. Sobre Colciencias. Disponible desde Internet en: http://www.colciencias.gov.co/colciencias/sobre-colciencias (con acceso 22/05/2017).

4. COLCIENCIAS. 2016a. Reconocimiento de Investigadores del Sistema Nacional de Ciencia, Tecnología e Innovación. Publicación de resultados finales de la convocatoria 737 de 2015. Disponible desde Internet en: http://www.colciencias.gov.co/sites/default/files/ listado-publicacion-resultadosfinales-conv737-investigadores-consulta.pdf (con acceso 22/05/2017).

5. COLCIENCIAS. 2016b. Reconocimiento y Medición de Grupos de Investigación, Desarrollo Tecnológico o de Innovación. Publicación de resultados finales de la convocatoria 737 de 2015. Disponible desde Internet en: http://www.colciencias.gov.co/sites/default/ files/upload/paginas/conv-medicion-737-2015.pdf (con acceso 22/05/2017).

6. U.D.C.A. 2017. Misión. Universidad de Ciencias Aplicadas y Ambientales U.D.C.A. Disponible desde Internet en: http:/www.udca.edu.co/informacion-institucional/ (con acceso: 22/05/2017).

7. PEI. 2013. Proyecto Educativo Institucional-PEI-. Disponible desde Internet en: http://www.udca.edu.co/ wp-content/uploads/2015/02/Proyecto-EducativoInstitucional.pdf (con acceso 22/05/2017). 